KANSAS JOURNAL of MEDICINE

\section{Retained Mediastinal Contrast}

Omar A. Almoghrabi, M.D., Gregory F. Muehlebach, M.D. University of Kansas Medical Center,

Department of Cardiothoracic Surgery, Kansas City, KS Received Oct. 25, 2019; Accepted for publication Dec. 16, 2019; Published online March 20, 2020

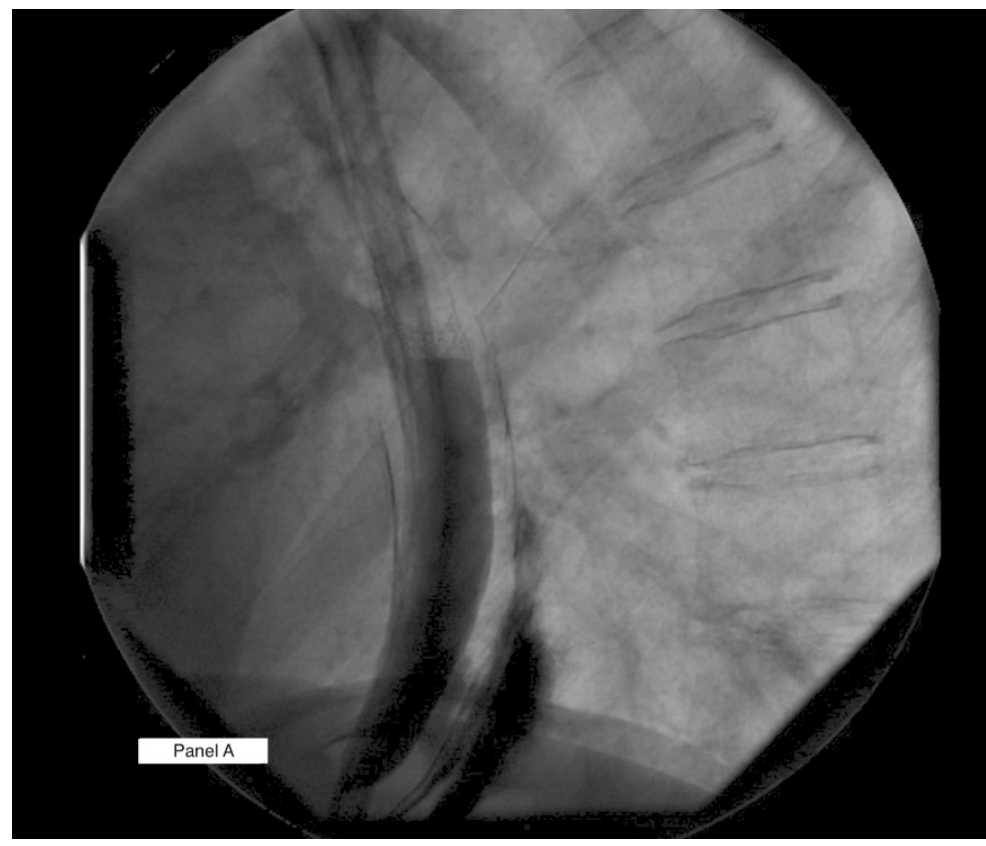

Figure 1. Barium swallow study from 2006 demonstrating non-communicating extraluminal contrast within the mediastinum.

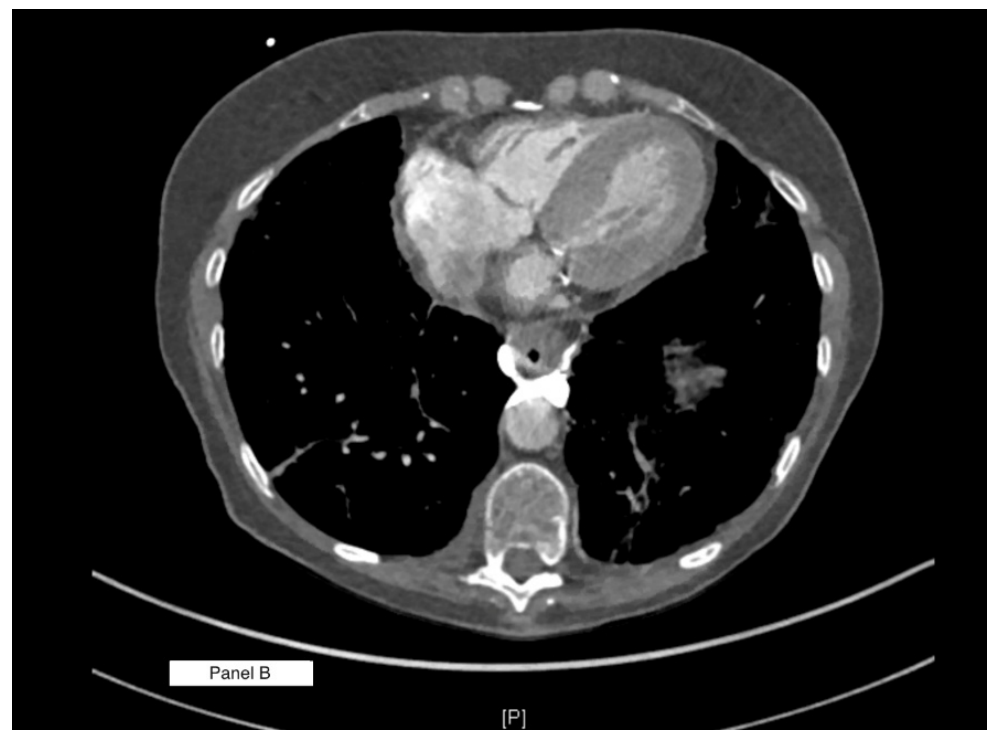

Figure 2. Axial computed tomography image from 2019 demonstrating noncommunicating extraluminal contrast within the mediastinum.

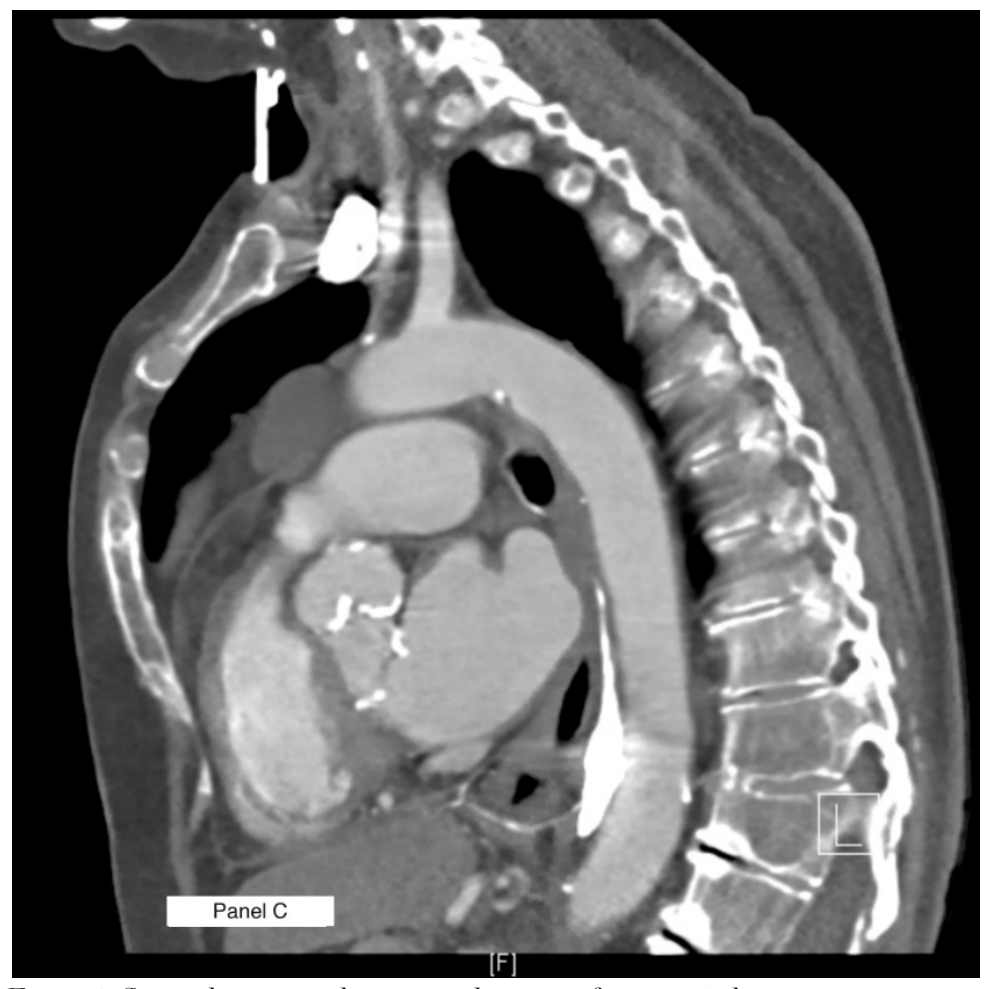

Figure 3. Sagittal computed tomography image from 2019 demonstrating noncommunicating extraluminal contrast within the mediastinum.

\section{CASE DESCRIPTION}

A 79-year-old female is presented with history of total laryngectomy, placement of tracheoesophageal puncture for phonation, and radiation therapy secondary to laryngeal cancer in the year 2000 . Over the course of 2004, she developed progressive dysphagia due to esophageal strictures requiring a number of dilation procedures with the complication of esophageal perforation in late 2005. The perforation was treated conservatively, however, her esophageal strictures became refractory to dilation. In 2006, the patient underwent a barium swallow study for diagnostic purposes and subsequently had multiple x-rays and computed tomography (CT) scans demonstrating pre-existing non-communicating extraluminal contrast, likely from her known esophageal perforation in late 2005 (Figure 1). Following those scans, she underwent reconstruction of the pharyngoesophageal stricture with tubed left radial forearm free flap. In 2019, she presented for aortic valve replacement work-up and underwent a CT scan with findings initially puzzling to the medical team. The scan demonstrated the retained contrast 13 years later (Figures 2 and 3). The patient did not exhibit any systemic signs of infection or dysphagia.

\section{DISCUSSION}

This unprecedented finding has not been reported in the literature previously. Although the patient has not had any clinical sequelae secondary to the retained barium contrast, long-term effects remain unknown. This finding demonstrated the importance of utilizing an absorbable water-soluble contrast as a first step for the diagnosis of perforated viscus given the serious implication of non-absorbable barium contrast and resultant inflammatory reaction. ${ }^{1,2,3}$ However, in a contained rupture, as in this case, barium contrast did not elicit mediastinitis and the patient remained asymptomatic from it. 


\section{REFERENCES}

${ }^{1}$ Buecker A, Wein BB, Neuerburg JM, Guenther RW. Esophageal perforation: Comparison of use of aqueous and barium-containing contrast media. Radiology 1997; 20(3):683-686. PMID: 9051016.

2 Grobmyer AJ 3rd, Kerlan RA, Peterson CM, Dragstedt LR 2nd. Barium peritonitis. Am Surg 1984;50(2):116-120. PMID: 6703517.

${ }^{3}$ Gollub MJ, Bains MS. Barium sulfate: A new (old) contrast agent for diagnosis of postoperative esophageal leaks. Radiology 1997; 202(2):360-362. PMID: 9015057.

Keywords: contrast media, laryngeal cancer, esophageal diseases
KANSAS JOURNAL of MEDIC INE

RETAINED MEDIASTINAL CONTRAST continued. 